\title{
Tap-Changing Operations of Multiple Transformers using ANN Based Controller
}

\author{
Md Fakhrul Islam \\ Faculty of Sciences, Engineering \& Health \\ CQUniversity, Rockhampton, Australia \\ Email: Fakhrulislamfa@gmail.com \\ Amanullah Maung Than Oo \\ Faculty of Sciences, Engineering \& Health \\ CQUniversity, Rockhampton, Australia \\ Email: a.maungthanoo@cqu.edu.au \\ Received 21 September 2011 \\ Accepted 17 November 2012
}

\begin{abstract}
Tap changing operations of multiple transformers at a substation is a complex problem due to issues in controlling the parallel operations of the transformers involved, differences in manufacturer's design and inaccuracy of the measures. In earlier work Artificial Neural Network (ANN) based tap changer controller has been developed to overcome these difficulties and improved results have been shown for systems based on two parallel transformers. This paper aims to improve the previously proposed ANN controller configuration to suit for multiple transformers. This new configuration of ANN controller uses ensemble technique and illustrates the operational efficiency. In particular, it has been shown that the ANN controller designed for two parallel transformers in this new technique will operate for multiple transformers. This means that if additional transformers (more than two) are installed in a substation to cope up with increased load, the ANN controller needs not to be redesigned/replaced.
\end{abstract}

Keywords: ANN, Tap-changer, Ensemble, Transformers.

\section{Introduction}

In power transmission and distribution system, transformers with built-in on load tap changing mechanism play a vital role in maintaining voltage level by changing their tap position. In order to avoid complete failure of supply for any fault of one line or transformers, distribution transformers are arranged to operate in parallel using circuit breakers. This parallel operation of transformers has many other advantages such as maintenance and major repair works. In this paper, the transformers in the substation are assumed to be operating always in parallel. With the increasing demand of the electricity, substations are frequently upgraded. This upgrading process usually requires the replacement or modification of many existing control circuits and devices to adjust with the new machinery. Also, the adjustment needs to cope with the variations of models due to different manufacturers. ANN based tap changer controller was developed Islam et al. (2005) to overcome the difficulties of existing controllers (E. T. Jauch, 2001, Okanik et al., 1999, J. H. Harlow, 1996, Thornley and Hiscock, 2001) in complex substation configuration. In the related earlier works (Islam, 2006, Islam et al., 2005) efficacy of ANN learning algorithm to address the issues, arising in regards to tape changer control, have been demonstrated. But, the focuses of these works have been on systems consisting of two transformers. This paper presents a new ANN based tap-changer control for more complex systems, consisting of multiple transformers. We show that the developed approach effectively deals with the complex tap changer operations for multiple transformers. So the new ANN based tap controller can be used to replace the hardware based circuits. Also the new controller 
allows the flexibility of adding transformers to meet the increasing load demand without any replacement.

The rest of the paper is organized as follows. Section 2 briefly conveys the previous work. Section 3 highlights the structure of ANN based tap changer. Section 4 then discusses on the data used in our experiments, with the next section (Section 5) presenting the experimental results and discussion. Section 6 highlights the model selection and some implications of using tap controller with increased number of transformers. Section 7 contains recommended changes in initial design of tap controller. Section 8 concludes the paper.

\section{Previous Relevant Work}

ANN has been used in applications of various domains including industrial operation (Saha et al., 1998), manufacturing (Kamruzzaman et al., 2006) healthcare (Begg and Kamruzzaman, 2003), and finance (Kamruzzaman and Sarker, 2003). The use of ANN in tap changer control was initiated by a research undertaken by the first author (Islam, 2006). Structural design which is known as the architecture of an ANN consists of neurons at the input layer, hidden layer and output layers with interconnections between the neurons in the different layers. Fig. 1 shows an artificial neural network for seven input variables, four hidden and single output neurons.

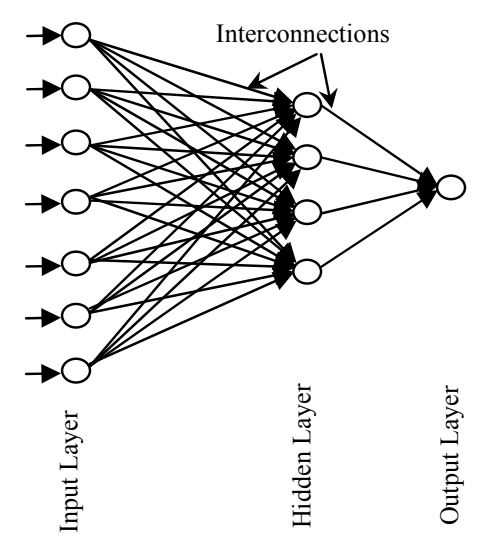

Fig. 1: An Artificial Neural Network for seven input variables, four hidden and one output neurons
Interconnections own weights that manipulate the data in the calculation from input through hidden units to output. The learning process for updating the weights of the interconnections for particular application is performed by learning algorithm.

A set of existing ANN learning algorithms like Bayesian Regularization back propagation (BRBP) (Foresee and Hagan, 1997), Scaled Conjugate Gradient (SCG) (Moller, 1993), and Cascade-correlation (CC) (Fahlman and C. Lebiere, 1990) learning algorithms were chosen due to their recognized good performance in wide range of applications (Bishop, 1995a).

Among these algorithms in back propagation the weights and biases are adjusted by negative of the gradient descends to minimize performance function. SCG uses some variation techniques such as conjugate gradient. The cascade correlation algorithm, during training process, adds hidden unit one at a time and tries to minimize residual error and maximize the correlation between the new unit's output and the residual error signal of the network.

Input variables were selected based on well understood factors influencing the tap changer control (Beckwith Electric Co, 1999, Harlow, 1996). These were the power system measuring components such as voltage level, reactive powers, real power, power factor, circulating current and the status of circuit breakers involved in transformers' parallel operation (Islam et al., 2004a). Real world data was obtained from an Australian electric supply company. The measurements were recorded under normal operating conditions. Some of the data, that were hardly available from the power supply company, were mathematically derived from the power network parameters supplied by the company (Islam et al., 2004a, Islam et al., 2003).

In regards to performance this paper identifies the best performance of model on the basis of the highest number of recognition or minimum number of misrecognition (False response) where MSE and STD exhibit reasonable lower values from test data. So, out of multiple trials, the best performance for ANN trained by the BRBP was $98.41 \%$ correct recognition, while that for ANN trained using SCG was $98.3 \%$ correct recognition (Islam et al., 2004a). Both of these results were achieved for ANN architecture with 4 hidden 
units. For ANN trained using cascade-correlation algorithm, the best performance was $98.9 \%$ correct recognition (Islam et al., 2004b).

During training $\mathrm{CC}$ algorithm builds network architecture from smallest (one hidden unit) to a required size as well as updates the weights to provide correct outputs. It is found that the best performing ANN, trained using $\mathrm{CC}$ algorithm, created architecture consisting 4 hidden units and it did not converge (i.e. fails to improve the correlation scores) at less than that number of hidden units.

Since the distribution transformers in power system play a vital role in voltage regulation and system stability, it is always necessary to have better performance in tap changing operation. With this view, modification of cascade-correlated learning algorithm was proposed in (Islam et al., 2005, Islam, 2006) where learning took places by minimizing an objective function that minimizes the squared error at the output, smoothes the weights and maximizes the correlation co-efficient between new unit's output and the residual error signal. ANN, trained using this new algorithm, improved the best performance to $0 \%$ misrecognition $(100 \%$ correct recognition) with the achievement of architecture size of 2 hidden units, less than the previously existing algorithms (Islam, 2006).

All these stated experiments and developments were carried out concentrating on two parallel transformers only and avoided the complicacy in training ANN when multiple transformers are considered. In this contribution, we propose an extension to these previous developments and focus on the situations when more than two transformers are added in the substation.

\section{Proposed ANN Based Transformer Tap Changer Control}

A single line diagram of four transformers, operating in parallel and using the proposed ANN based tap controller, is shown in Fig. 2. The ANN based tap controllers receive signals from the power system and decide about the tap raise, tap lower and tap hold. The decision is then conveyed to the tap changing mechanism using a relay for execution of the tap changer operations. The proposed ANN based controller can be implemented in two ways. It can be implemented in hardware or as a software program.

Algorithms are used to train the ANN model i.e. architecture and weights of the interconnections. Performance of the algorithms is measured how far they could update the weights of the architecture to provide the correct outputs of the unseen input data. Once it is finalized the model (i.e., weights, biases, nodes and other parameters) can be embedded as chips or used as software in the computer.

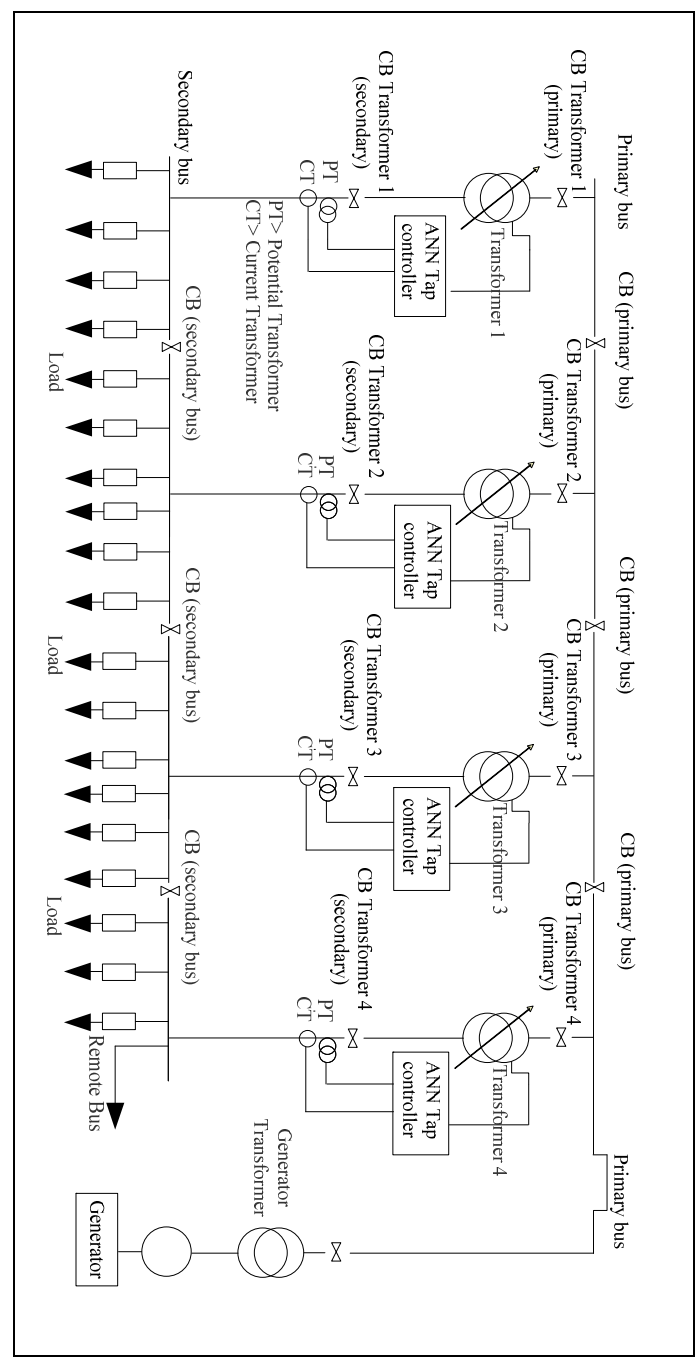

Fig. 2: Single line diagram of four transformers in substation with ANN based tap changing controller

For implementation in a hardware system neural network needs the support of inter processing 
communication and memory devices along with the function of individual processing elements. In this perspective a Very Large Scale Integration (VLSI) Processor with input/output device, Erasable Programmable Read Only Memory (EPROM), that contains executable instructions and an operating system on a single board can be used to implement an ANN module. Fig. 3 shows the schematic diagram of such a microprocessor control ANN device.

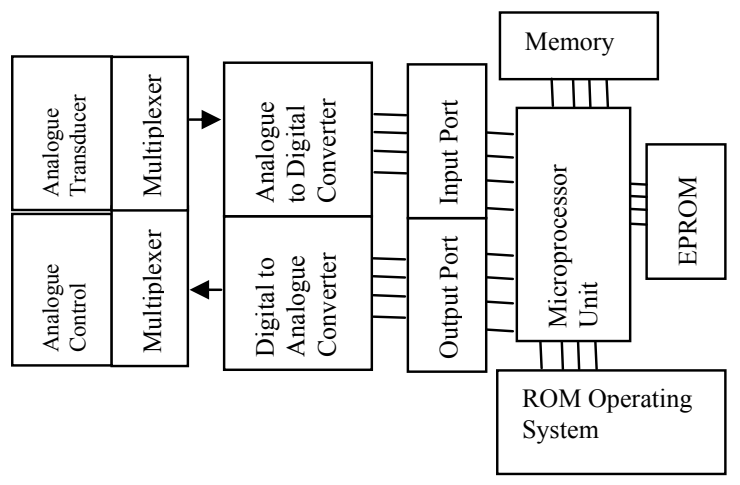

Figure 3: Schematic diagram of on-line chip for ANN tap changer controller

Input signals obtained from the power system through analogue transducer can be collected onto a single highspeed line using a multiplexer and fed into the analogue to a digital converter. The digital information from the analogue digital converter is then fed into the microprocessor through the input port. The operating software program controls the activation of the ports to intake signals, runs the neural network module, and delivers the microprocessor's results. The analogue control signal is obtained at the output in the reverse manner of the input signal and finally conveyed to the tap changer operating mechanism. In this method of implementation any new advances in ANN simulation algorithm can be implemented by erasing and reprogramming the EPROM. Alternately, a configured ANN architecture, the computed weights and node transfer function can be directly implemented in a VLSI chip with built in microprocessor units. This type of control can only be used for fixed functionality that that means the implemented, weights and node's function on chip cannot be changed which needs replacement if an upgrading is required in the system.

More detail about implementation of the ANN model into hardware and software uses are beyond the scope of this paper. This paper mainly deal with the development of configuring the ANN model (architecture and weights determination) to provide better results i.e. accurate outputs for unseen test data and technical analysis to support the improvement occurred.

In power systems, a centralized control system termed as SCADA (Mavrin et al., 1999) is used for control and data acquisition. The system allows the ease of using software based system through a set of application servers. This proposed design of control system can also be used in SCADA system as software based implementation in its server. But, huge number of distribution transformers tap changer is controlled locally for voltage regulation in distribution substations. So, we propose a design change in the tap changer control system that uses hardware based ANN implementation at the local substations.

Naturally, if ANN based tap controller is implemented as hardware, then the replacement will add some cost. The more the numbers of substations need to be upgraded, the more the cost will be added. However, if careful steps are taken into consideration during the initial implementation design, replacement of the hardware implemented ANN based controller can be avoided even when the number of transformers is increased. This is where the key contribution of this work comes in. We investigate an ANN based tap changer control system, that even if being trained on two transformers, can readily be used for three or even more transformers.

\section{Data}

The power network, transformer details, and load data variations supplied by Australian Electric Supply Company are used for this experiment with the number of operating transformers being up to four in parallel. Each of the 150 MVA transformers has 10 tap positions and is capable of $17.55 \%$ voltage variation at a step of $4.29 \mathrm{kV}$. The transformer percentage variation is from $14.9 \%$ to $22.7 \%$. Although transformers are loaded lightly (below their capacity), the maximum load of 300 MVA per transformer is considered in data generation. Data consists of numerical values of voltage level, circuit breaker status, real power and reactive power of the individual transformers, as well as, total real power and reactive power of the transformers operating in 
parallel and the circulating currents when the tap position differs between transformers. Numerical values of these variables at a particular moment are grouped to form a vector ' $\mathbf{x}$ '. Data set is formed by a set of vector ' $\mathbf{x}$ ' in such a way that it covers all possible variations of the voltage levels, circulating currents for tap position differences, real and reactive power variations, and circuit breaker status.

Three such data sets are produced considering parallel operations of two transformers, three transformers and four transformers. They are named as DM2Tx, DM3Tx, and DM4Tx respectively. Each of the data set has three types of data for three operations of transformer tap: tap raise, tap hold and tap lower. In the produced data sets, each of these types is associated with a target vector as follows.

$$
\begin{array}{ll}
\mathbf{t}_{1}=[-1,1,1] & \text { tap raise } \\
\mathbf{t}_{2}=[1,1,-1] & \text { tap lower } \\
\mathbf{t}_{3}=[1,-1,1] & \text { tap hold }
\end{array}
$$

Each of the data sets (DM2Tx, DM3Tx, and DM4Tx) is divided into two subsets: one for training and another for testing. Stratified sampling (Arvo, 1995, Stehman, 1996) is used to generate the training and test subsets from the types of raise, lower and hold.

\section{Experiments, Results and Discussion}

ANN classification process is applied on the produced data. In this target association, the output of the ANN has three neurons. For a particular tap operation, the value of a neuron will be ' -1 ', while the other neurons' values will be ' 1 ' (i.e., values corresponding to the target vectors assigned in the dataset). The neuron with the value of ' -1 ' will guide the tap changing operations. For instance, if a test data, presented to the trained ANN, triggers the first output neuron value to be '-1' (or close to ' -1 '), a signal is transmitted to a certain relay that operates the tap changing mechanism for raising the position. Similarly, if third output neuron has the value of ' -1 ' (or close to ' -1 '), signal is transmitted to another relay that operates the mechanism to lower the tap position. In the same way, if the second neuron, has the value of ' -1 ' (or close to ' -1 '), it will guide the relay that locks the circuit to keep tap in the same position.

During training, the ANN connection weights are setup in such a way that the respective type of data provides the ' -1 ' (or close to ' -1 ') value to the relevant neuron. Three models are built up from training the three data sets (representing the different number of transformers in operations). Modified Cascade Correlation algorithm (MCC), developed particularly for the tap changer control (Islam et al., 2004b, Islam, 2006), is used to train the models. Since the algorithm is an efficient trainer of the ANN model for tap changer control, 15\% of the data are used for training and the rest are used for testing.

Requirement of training data is completely solutiondependent. The most important point of selecting training data in this project is that the sample of the training data set should cover operations of the tap changer within the entire tap position range to maintain the correct voltage level. This has been ensured in selecting the $15 \%$ of the training data set. So, more than those $15 \%$ were not necessary and were not taken to maintain minimum training time.

For each of the transformer settings, thirty trials are performed to produce a series of 30 trained ANNs. The produced models are labeled as follows.

- M2Tx represents the series of models trained with DM2Tx (two transformers in operation).

- M3Tx represents the series of models trained with DM3 Txt (three transformers in operation).

- M4Tx represents the series of models trained with DM4Tx (four transformers in operation).

MCC algorithm, automatically determines the number of hidden units and architecture of the ANN during training. Table 1 shows the numbers of hidden units at ANN learning convergence for the 30 trials in our experiments.

Table 1: The numbers of hidden units (HU) at ANN training convergence for the 30 trials

\begin{tabular}{lrrrrrr}
\hline $\begin{array}{l}\text { Model } \\
\text { series }\end{array}$ & HU2 & HU3 & HU4 & HU5 & HU6 & HU7 \\
\hline M2Tx & 22 & 3 & 1 & 1 & 1 & 2 \\
M3Tx & 19 & 2 & 2 & 1 & 1 & 5 \\
M4Tx & 11 & 5 & 1 & 3 & 3 & 7 \\
\hline
\end{tabular}

Among the models created by MCC algorithm with less than seven hidden units in 30 runs met the set criteria based on the objective function and ended the training. 
So, they are ensured as well trained. The models created with seven hidden units could not meet the set criteria of objective functions and the training process was terminated because of reaching the highest epoch number. So, they are not considered well trained and do not bear any interest.

Smaller ANN architecture has been shown to offer better generalization capability by previous researchers (R. Reed, 1993) in various areas. Smaller architecture means less number of hidden units since hidden units determine the size of the architecture in this project. MCC algorithm, automatically determines the number of hidden units and hence the architecture of the ANN during training.

It is observed that maximum number of the ANN trials have converged with two hidden units for all the transformer settings It is further observed that the number of trials converging with two hidden units (HU2) decrease with the increased number of transformers. In the previous work (Islam, 2006), which was carried out for two transformers, the existing $\mathrm{CC}$ algorithm fails to converge below four hidden units and also the other algorithms mentioned above, could not train well the ANN architecture with four hidden units to provide better performance. . In this regards it can be stated that the existing MCC algorithm is better trainer of ANN based transformers' tap changer controller.

\section{Selection ANN Model for Application}

A trained ANN has an inherent property of generalization by which it can estimate new unseen inputs and produce output accurately (Bishop, 1995b, Zhang et al., 2003). Also, this inherent property of ANN generally governs its performance capabilities. The different error statistics on test data, provide a good indication of ANN's generalization as well as performance capabilities.

As the smaller ANN models exhibit better performances (R. Reed, 1993) this paper will consider the ANN models with two hidden units in all the subsequent evaluations and uses three criteria in analysing the performances of the models. These are false response rates, mean squared error (MSE) and standard deviation (STD).

\subsection{Options M2Tx, M3Tx or M4Tx as per number of transformers}

Among the different trained ANN models, it is important to select an ANN model for practical use. For this, it is necessary to verify the performance capability of the models. The false response rate versus the trained ANNs sorted in ascending order of false response rate is shown in Fig. 4.

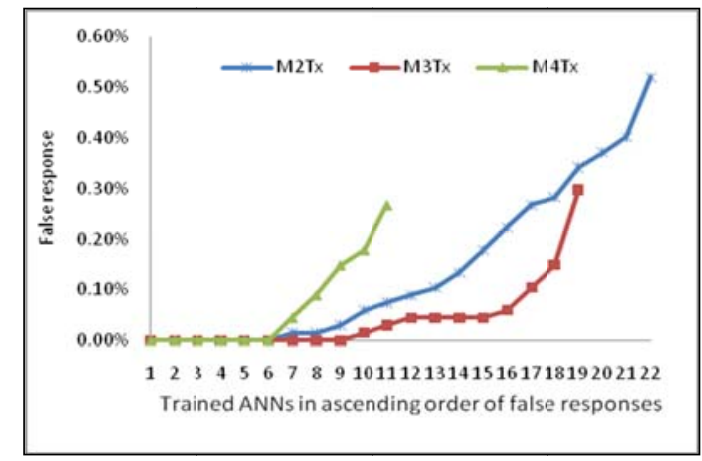

Fig. 4: The false response rate versus the ANN trials in ascending order of false response rates

It has been observed that the training algorithm build nine models in M3Tx series that provide zero percent false responses (i.e. 100\% correct response) on the test data. Each of the other Model series (M2Tx and M4Tx) has six models with zero percent false responses.

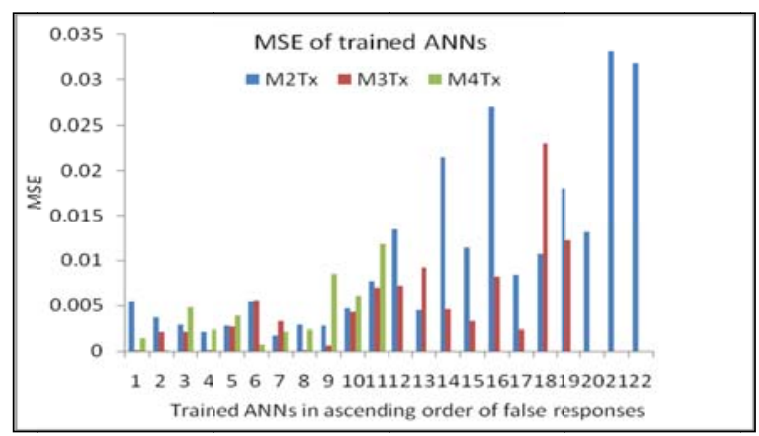

Fig. 5: Mean square errors versus trained ANNs in ascending order of false responses

MSE shows how close the ANNs predicted values are to the desired target values. If MSE is small for the test data, the ANNs are assumed to have good performance capability. Fig. 5 shows the Mean Squared Error (MSE) versus the trained ANNs sorted in ascending order of false response rate. It shows that the MSE varies even 
when the trained models possess zero percent false responses on test data.

Standard deviation shows how the estimated values vary from their mean. So, models with low standard deviation are expected to increase the probability of correct recognition of the new similar data. The standard deviations of the ANN models are given in Fig. 6. It shows that the STD varies even when the models acquire zero percent false responses. In Fig. 4 and Fig. 5 it can be observed that although some of the ANNs produce exactly the same number of false response rates in different trials, they differ in MSE and STD. Thus it is important to consider all the three measures in selecting ANN model among the trials.

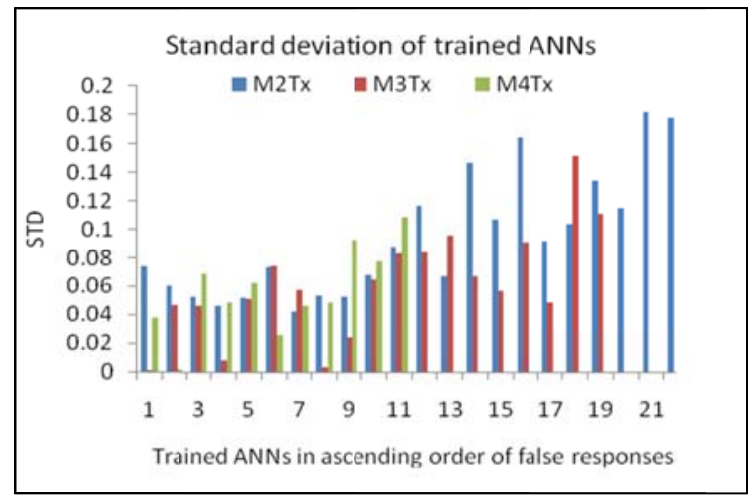

Fig. 6: Standard deviation versus trained ANNs in ascending order of false responses

A number of models in M3Tx series offer same nonzero false response rate, which is close to the average value among the trials. But they differ in both the MSE and STD values. Overall, it is observed that ANNs rarely achieve identical performance among the all trials. This is due to the dependency on initial weights, local minima and other factors. ANN training uses gradient descent technique to minimize the error between output and targets. The surface of this error has numerous minima's. The one has the lowest value is called global minima which is the goal of the training process to reach. Others are called local minima. Choice of initial weight may happen to be the neighbour of a local minima and the training process may dictate towards that local minima and converge at that point or its vicinity. That is the reason ANN algorithms are run several times to find the one which terminated nearest to the global minima to acquire the quality solution.
Quality of the ANN converged to local minima varies according to the depth of the local minima relative to the global minima. In the experiments, however, the best, average and worst performing ANN models were selected considering all the three measures. Table 2 presents the false response rates for the transformer networks. Fig. 7 and Fig. 8 show the corresponding MSE and STD values respectively.

Table 2: False responses of best average and worst ANN models with two hidden units in the three model series

\begin{tabular}{llll}
\hline $\begin{array}{l}\text { Model } \\
\text { series }\end{array}$ & \multicolumn{2}{l}{$\begin{array}{l}\text { Percentage of false responses for test } \\
\text { data }\end{array}$} \\
\cline { 2 - 4 } & Best & Average & Worst \\
\cline { 2 - 4 } M2Tx & 0.0 & 0.13 & 0.52 \\
M3Tx & 0.0 & 0.04 & 0.30 \\
M4Tx & 0.0 & 0.04 & 0.27 \\
\hline
\end{tabular}

As per the results in Table 2, the false responses remain equal for the best performing models irrespective of the number of transformers. But for average and worst performing models, false responses reduce for more than two transformers.

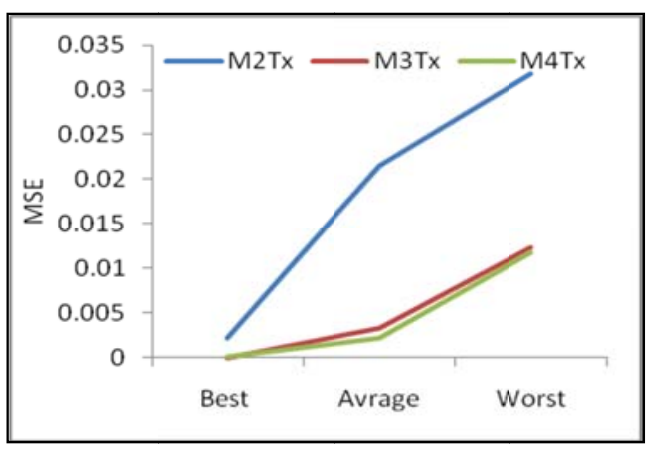

Fig. 7: Mean Squared Error (MSE) of best average and worst ANN models with two hidden units in the three model series

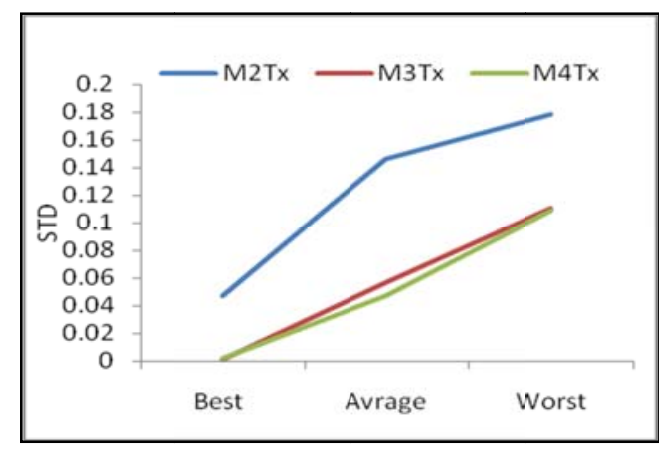

Fig. 8: Standard deviation (STD) of best average and worst ANN models with two hidden units in the three model series 
MSE in Fig. 7 and STD in Fig. 8 show similar variations as false responses. Both the MSE and STD values reduce for more than two transformers. Further, the MSE and STD for both the M3Tx and M4Tx are close, indicating that their performance capabilities are approximately equal.

Generally in ANN based applications, the model achieving near to average performance is chosen. Reason behind this phenomenon is, the best performing model is often assumed to have less generalization capability than the average model. However, in this experiment, $15 \%$ of the total data are used for training the models and remaining $85 \%$ are used for testing the models. So, the models showing small false responses imply that they recognize accurately large number of unseen test data (for the relevant number of transformers). Thus, the best performing models are considered to have high generalization capacity in this experiment and are chosen from the model series for implementation.

\subsection{Implications Using M2TX Model with increased Number of Transformers}

In the previously discussed control system, the model M2Tx is used when two transformers are operating in a substation. For a substation upgrade (to three or four transformers), ANN requires to be retrained and, as mentioned in Section 3, this imposes additional costs.

To explain the issue further, this section shows the consequences of using the same M2Tx model for increased number of transformers. In other words, Model M2Tx are presented the test data DM3Tx (for three transformers) and DM4Tx (for four transformers), and the response of M2Tx are placed into Table 3.

Table 3: Percentage of false responses by model M2Tx when transformers are increased

\begin{tabular}{cccc}
\hline $\begin{array}{c}\text { Number of } \\
\text { transformers } \\
\text { operating in } \\
\text { Parallel }\end{array}$ & \multicolumn{3}{c}{ Percent of false responses } \\
\cline { 2 - 4 } & Best & Average & Worst \\
2 & 0.0 & 0.13 & 0.52 \\
3 & 0.06 & 0.56 & 1.99 \\
4 & 0.07 & 0.92 & 2.44 \\
\hline
\end{tabular}

Fig. 9 and Fig. 10 shows the MSE and STD respectively. Experimental results show that the continued use of M2Tx model, for a substation upgrade from two to more than two transformers, results in increase of false responses. MSE and STD also increase, as observed in Fig. 9 and Fig. 10. The next section will show an improvement to this model.

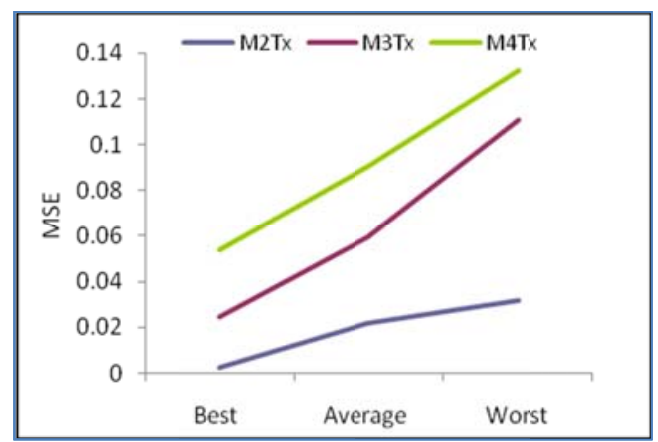

Fig. 9: Mean square error of model M2Tx when transformers are increased

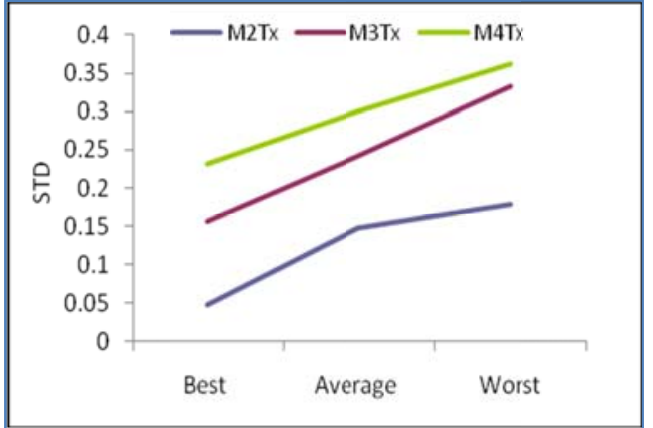

Fig. 10: Standard deviation of model M2Tx when transformers are increased

\section{Ensemble Model in Initial Design to Fit More Transformers in Future}

This section proposes an ensemble of ANNs, trained on using two transformers only (M2Tx model), that shows performance improvement even when the substation is upgraded from two to three or four transformers. It has been observed that estimation of same data at the output by different ANN models is not identical. This is due to the fact that one model may set output very close to the target value, whereas another model may set output far to the target value. For another data point, these characteristics may vary considerably. In these situations, ensemble technique may perform well and this section investigates the effectiveness of ensemble of 
ANN. ANN ensemble is formed by combining two or more different trial outcomes (models) as in Fig. 11.

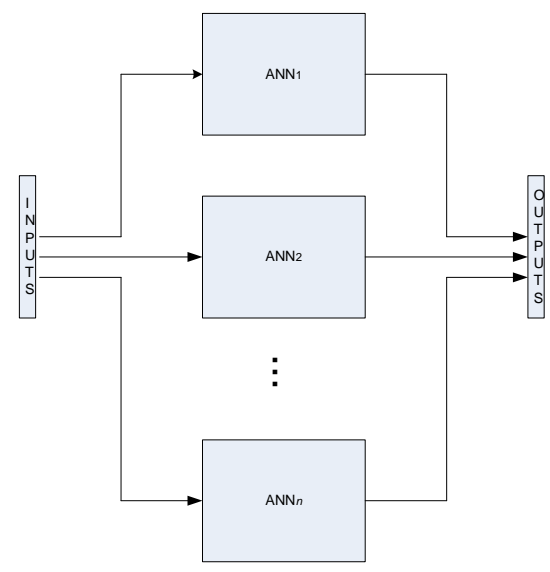

Fig. 11 An ensemble of $n$ numbers of trained ANNs

The effectiveness of the formed ensembles depends on how these models are chosen ((Ed.), 1999). In reference to the Fig. 11, which shows an ensemble of $n$ numbers of trained ANNs, let the output $\left(y_{\mathrm{e}}\right)$ of the ensemble is taken as the average of the ANN members' outputs as expressed in (1).

$$
y_{e}(\mathbf{x})=\frac{1}{n} \sum_{i}^{n} y_{i}(\mathbf{x})
$$

where $n$ represents the number of trained ANNs, $y_{\mathrm{i}}(\mathbf{x})$ is the output of $i^{\text {th }}$ trained ANNs where $i=1$, $2, \ldots, n$. In that case, error due to ensemble is given as

$$
\begin{aligned}
& E_{e}=\varepsilon\left[\left(\frac{1}{n} \sum_{i}^{n} y_{i}(\mathbf{x})-t\right)^{2}\right] \\
& =\varepsilon\left[\left(\frac{1}{n} \sum_{i}^{n} e_{i}\right)^{2}\right] \\
& =\frac{1}{n} \varepsilon\left[\left(\sum_{i}^{n} e_{i}\right)^{2}\right] \\
& =\frac{1}{n} E_{\text {avg }}
\end{aligned}
$$

where $\varepsilon$ [.] denotes the expectation and $E_{\text {avg }}$ is the average error of the ANN members. Equation (2) explains that the sum of square error of the ensemble ANNs is reduced by $n$ when the output is determined by averaging the output of member ANNs. As per the
Cauchy's inequality, as shown in Equation (3), the averaging process guarantees not to increase the error.

$$
\left(\sum_{i}^{n} e_{i}\right)^{2} \leq n \sum_{i}^{n} e_{i}^{2}
$$

Hence, the ensemble error is less or qual than the average error in members as in Equation 4.

$$
E_{e} \leq E_{\text {avg }}
$$

In this experiment, the ensemble based control model is formed by the best and average models as members and the above averaging process is applied.

Results for test data sets DM3Tx (three transformers in parallel) and DM4Tx (four transformers in parallel), as well as DM2Tx (two transformers in parallel), are shown in Table 4. The first two columns show the performance for best (member 1) and average model (member 2), while the last column shows the outcome for ensemble.

Table 4: Percentage of false responses by Ensemble model (M2Tx) when number transformers are increased

\begin{tabular}{cccc}
\hline $\begin{array}{l}\text { Number of } \\
\text { transformers } \\
\text { operating in } \\
\text { Parallel }\end{array}$ & \multicolumn{3}{l}{ Percent of false responses } \\
\cline { 2 - 4 } & $\begin{array}{l}\text { Ensemble } \\
\text { member 1 } \\
\text { (M2Tx) }\end{array}$ & $\begin{array}{l}\text { Ensemble } \\
\text { member 2 } \\
(\mathrm{M} 2 \mathrm{Tx})\end{array}$ & $\begin{array}{l}\text { Ensemble } \\
\text { Model } \\
(\mathrm{M} 2 \mathrm{Tx})\end{array}$ \\
\cline { 2 - 4 } 2 & 0.0 & 0.13 & 0 \\
3 & 0.06 & 0.56 & 0 \\
4 & 0.07 & 0.92 & 0 \\
\hline
\end{tabular}

The results show that the ensemble improves performance by the reduction of false responses even with the increase of number of transformers. Thus, the same model (originally designed for two transformers) can be used for three or four transformers and allow easy upgrade of the substation.

\section{Conclusions}

In this paper, the performances of ANN models trained by existing famous algorithms and newly developed Modified Cascade Correlation algorithm for two to four numbers of transformers in parallel operation have been investigated. The paper also studied the limitations of parallel operation when transformers are increased to upgrade substation to take more loads but the tap changing controllers remained unchanged. The paper 
has developed an ensemble scheme and shows that the ANN based controller, configured following this scheme for two parallel transformers, will be suitable for multiple transformers in parallel operation. This means that installation of new transformers in substations, to meet increasing load demand in future, is possible without replacing the ANN based tap controller.

\section{References}

1. (ED.), A. J. C. S. 1999. Combining Artificial Neural Nets: Ensemble and Modular Multi-Net Systems, London; New York: Springer.

2. ARVO, J. Stratified sampling of spherical triangles. The 22nd annual conference on Computer graphics and interactive techniques 1995. ACM, 437-438.

3. BECKWITH ELECTRIC CO 1999. Advanced Paralleling of LTC Transformers by the Circulating Current Method, Tap changer Control Application. Note \# 13, Beckwith Electric Co., Inc.,.

4. BEGG, R. \& KAMRUZZAMAN, J. Comparison of neural network and support vectors machines for recognizing young-old gait patterns. IEEE TENCON 2003, 2003 India. 354-358.

5. BISHOP, C. M. 1995a. Neural Network for Pattern Recognition, New York, Oxford University Press Inc.

6. BISHOP, C. M. 1995b. Neural networks for pattern recognition, Oxford University Press, USA.

7. E. T. JAUCH. Advance transformer paralleling. IEEE PES Transmission and Distribution Conference and Exposititon, 2001.

8. FAHLMAN, S. E. \& C. LEBIERE 1990. The cascade-Correlation Learning Architecture. Advances in Neural Information Processing Systems, 2, 524-532.

9. FORESEE, F. D. \& HAGAN, M. T. 1997. Gauss-Newton approximation to Bayesian regularization. Proceedings of the International Joint Conference on Neural Networks, 1930-1935.

10. HARLOW, J. H. 1996. Load Tap Changing Control. National Rural Electric Cooperative Association (NRECA), March 24.

11. ISLAM, M. F. 2006. Artificial Neural Network for parallel Operation of Power Transformer. PhD, Monash University.

12. ISLAM, M. F., KAMRUZZAMAN, J. \& G. LU. Transformer tap-changer control using artificial neural network for cross network connection. Third IASTED International Conference on Power and Energy Systems (EuroPES 2003), 2003 Marbella, Spain. 666-671.

13. ISLAM, M. F., KAMRUZZAMAN, J. \& G. LU 2004a. Transformer tap changing by data classification using artificial neural network. Proc. IEEE PES Power System Conference and Exposition, 1417-1422, New York.

14. ISLAM, M. F., KAMRUZZAMAN, J. \& G. LU 2005. Improved ANN based tap-changer controller using modified cascadecorrelation algorithm. Journal of the Advanced Computational Intelligence and Intelligent Informatics, 9, No. 3, 226-234.
15. ISLAM, M. F., KAMRUZZAMAN, J. \& LU, G. Modified cascade-correlation algorithm for improved transformer tap changing operation,. Joint 2nd International Conference on Soft Computing and Intelligent Systems, 2004b Yokohama, Japan. 16.

16. J. H. HARLOW. Load Tap Chnging Control. National Rural Electric Cooperative Association (NRECA), 1996.

17. KAMRUZZAMAN, J., BEGG, R. \& SARKER, R. 2006. Artificial Neural Networks in Finance \& Manufacturing. Idea Group Publishing (USA).

18. KAMRUZZAMAN, J. \& SARKER, R. 2003. Comparing ANN based models with ARIMA for prediction of forex rates. ASOR Bulletin, p.2.

19. MAVRIN, M., KOROMAN, V. \& BOROVIC, B. SCADA in hydropower plants. Proc. IEEE International Symposium on Computer Aided Control System Design, 1999. 624-629.

20. MOLLER, M. F. 1993. A Scaled Conjugate Gradient Algorithm for fast supervised learning. Neural Networks, 6 525-533.

21. OKANIK, P., KURTH, B. \& J. H. HARLOW. An Update on the Paralleling of OLTC Powers Transformers. IEEE Transmission Distribution Conference, 1999. 871-875.

22. R. REED 1993. Pruning Algorithm-A Survey. IEEE Transactions on Neural Networks, 5, 740-746.

23. SAHA, P., SHOIB, M. \& KAMRUZZAMAN, J. 1998. Development of a neural network based integrated control system of $120 \mathrm{ton} / \mathrm{h}$ capacity boiler. Computers \& Electrical Engineering, 24, no. 6, 423-440.

24. STEHMAN, S. 1996. Estimating the kappa coefficient and its variance under stratified random sampling. Photogrammetric Engineering and Remote Sensing, 62, 401-407.

25. THORNLEY, V. P. \& HISCOCK, N. J. Improved Voltage Quality through Advances in Voltage Control Technique. Seventh International Conference on Developments in Power System Protection (IEE Conference Publ.No.479), 2001. 355-358.

26. ZHANG, S., LIU, H., GAO, D.-T. \& WANG, W. Surveying the methods of improving ANN generalization capability. The Second International Conference on Machine Learning and Cybernetics, 2-5 November 2003 Xi'an. 1259-1263. 